ARTICLE

\title{
Handedness-dependent quasiparticle interference in the two enantiomers of the topological chiral semimetal $\mathrm{PdGa}$
}

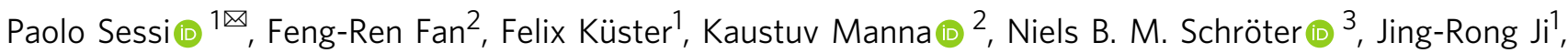
Samuel Stolz (1) 4,5, Jonas A. Krieger (1) 3,6,7, Ding Pei ${ }^{8}$, Timur K. Kim (1) 9, Pavel Dudin ${ }^{9}$, Cephise Cacho (1) ${ }^{9}$, Roland Widmer (10 ${ }^{4}$, Horst Borrmann (10 ${ }^{2}$, Wujun Shi ${ }^{10}$, Kai Chang ${ }^{1,11}$, Yan Sun (1) ${ }^{2}$, Claudia Felser ${ }^{2} \&$ Stuart S. P. Parkin (10) 1 四

It has recently been proposed that combining chirality with topological band theory results in a totally new class of fermions. Understanding how these unconventional quasiparticles propagate and interact remains largely unexplored so far. Here, we use scanning tunneling microscopy to visualize the electronic properties of the prototypical chiral topological semimetal PdGa. We reveal chiral quantum interference patterns of opposite spiraling directions for the two PdGa enantiomers, a direct manifestation of the change of sign of their Chern number. Additionally, we demonstrate that PdGa remains topologically non-trivial over a large energy range, experimentally detecting Fermi arcs in an energy window of more than $1.6 \mathrm{eV}$ that is symmetrically centered around the Fermi level. These results are a consequence of the deep connection between chirality in real and reciprocal space in this class of materials, and, thereby, establish PdGa as an ideal topological chiral semimetal.

\footnotetext{
${ }^{1}$ Max Planck Institute of Microstructure Physics, Halle 06120, Germany. ${ }^{2}$ Max Planck Institute for Chemical Physics of Solids, Dresden 01187, Germany. ${ }^{3}$ Swiss Light Source, Paul Scherrer Institute, CH-5232 Villigen PSI, Switzerland. ${ }^{4}$ EMPA, Swiss Federal Laboratories for Materials Science and Technology, 8600 Dübendorf, Switzerland. ${ }^{5}$ Institute of Condensed Matter Physics, Station 3, EPFL, 1015 Lausanne, Switzerland. ${ }^{6}$ Laboratory for Muon Spin Spectroscopy, Paul Scherrer Institute, CH-5232 Villigen PSI, Switzerland. ${ }^{7}$ Laboratorium für Festkörperphysik, ETH Zurich, CH-8093 Zurich, Switzerland. ${ }^{8}$ Clarendon Laboratory, Department of Physics, University of Oxford, Oxford OX1 3PU, United Kingdom. ${ }^{9}$ Diamond Light Source, Didcot OX110DE, United Kingdom. ${ }^{10}$ School of Physical Science and Technology, ShanghaiTech University, Shanghai 201203, China. ${ }^{11}$ Present address: Beijing Academy of Quantum Information Sciences, Beijing 100193, China. ${ }^{凶}$ email: paolo.sessi@mpi-halle.mpg.de; stuart.parkin@mpi-halle.mpg.de
} 
T he discovery of symmetry-protected topological materials represents a milestone in condensed matter physics ${ }^{1,2}$. They provide a new paradigm with regard to the band structure of solids, allowing for a classification of materials based on well-defined topological invariants that are calculated as global quantities from their bulk wave functions. On a fundamental level, the development of topological concepts in condensed matter has provided a fertile ground for the realization, in tabletop experiments, of concepts such as Majorana ${ }^{3,4}$ and Weyl fermions $s^{5,6}$, which were first predicted but never realized in the field of high-energy physics. More recently, it has been suggested that condensed matters systems can host totally new fermions, resulting from band crossings protected by specific symmetries of one of the 230 space groups $^{7}$. In this context, the concept of chirality occupies a primary role ${ }^{8-11}$. Chiral structures are characterised by a well-defined handedness due to the lack of both mirror and inversion symmetries, resulting in two distinct enantiomers. Their handedness can be manifested in several forms including non-collinear spin textures ${ }^{12}$, magnetochiral dichroism ${ }^{13}$, or unconventional superconductivity ${ }^{14}$. The additional existence of topologically non-trivial bands is expected to confer on chiral crystals unique physical properties which, not only don't exist in conventional materials, but are also forbidden in other topological classes ${ }^{11}$. These phenomena are directly linked to the Chern number, an integer used to classify the topological properties of the band structures in solids, and which is obtained by integrating the Berry curvature over a closed surface in momentum space. Because of the pseudovectorcharacter of the Berry phase, the Chern number reverts its sign under a mirror operation. Far from being a purely mathematical concept, this property has far-reaching implications and, in topologically non-trivial chiral crystals, is expected to result in radically new effects such as quantized circular photo-galvanic effects $^{15}$, unusual phonon dynamics ${ }^{16}$, and gyrotropic magnetic effects ${ }^{17}$.

Angle resolved photoemission (ARPES) studies have provided strong evidence for chiral Fermi arcs in chiral crystals belonging to the space group $P 2{ }_{1} 3$, number $198^{18,19}$. Recent scanning tunneling microscopy (STM) measurements detected surfaceorientation dependent states exhibiting chiral fermion characteristics in one CoSi enantiomer ${ }^{20}$. However, the experimental investigation of chirality-dependent phenomena, i.e., the emergence, observation, and manipulation of effects directly linked to the sign of the Chern number, remains largely unexplored. This requires chiral topological semimetals for which both enantiomers can be selectively synthesized so as to control the sign of the topological charge while keeping all other material properties unchanged ${ }^{21}$.

Here, we use STM to visualise how these unconventional quasiparticles propagate and interact with defects in the two enantiomers of the prototypical chiral semimetal PdGa. Our results provide compelling experimental evidence of a new and distinct feature of this class of materials: handedness-dependent scattering. We directly detect this effect in two distinct ways: (i) the opposite chirality of the quantum coherent interference patterns of the surface Fermi arcs in the two enantiomers and, (ii) their opposite energy-dependent spiraling direction, i.e., clockwise and anticlockwise. By imaging the perturbation pattern developing around defects in the crystal lattice of the two enantiomers, our results provide self-consistent experimental evidence of the deep connection between chirality in real and reciprocal space in this class of materials, with chiral multifold-fermion crossings in reciprocal space being protected by the chiral crystal structure in real space. Finally, by spectroscopically analysing unoccupied states, which are not accessible by conventional photoemission techniques, we demonstrate that PdGa remains topologically non-trivial over a very wide energy window, with Fermi arcs existing in an energy window of more than $1.6 \mathrm{eV}$ symmetrically centered around the Fermi level. These observations, jointly with the large extension of the Fermi arcs over the surface Brillouin zone, set PdGa as an ideal topological conductor, making it a promising platform to access and utilize optical and transport phenomena dictated by topology.

\section{Results}

Crystal structure and electronic properties. PdGa belongs to the family of chiral crystals with a cubic B20 structure, as illustrated in Fig. 1a. Gray and blue correspond to Pd and Ga atoms, respectively. The chirality can be distinguished by the handedness of the helix such as that formed by the Ga atoms, which rotates either clockwise or anticlockwise, depending on the enantiomer. The structural chirality directly determines the electronic properties. As schematically illustrated in Fig. 1b, symmetry-protected band crossings are visible at the $\Gamma$ and $R$ high symmetry points in the bulk. In particular, a spin-3/2 fermion is realized near the $\Gamma$ point, while a double spin-1 fermion is realized at the $\mathrm{R}$ (see Supplementary Note 1 for a detailed discussion). These crossings act as a source (red dot) or a sink (blue dot) of Berry curvature. They correspond to a Chern number of magnitude 4, i.e., the maximum Chern number achievable at a multifold node crossing $^{22}$, and reverse sign under a mirror operation ${ }^{23}$. Note that, contrary to non-chiral topological semimetals, these crossings are well-separated in energy. Because of the surface-bulk correspondence characterizing topological materials, this scenario results in the emergence of topologically protected surface Fermi arcs emanating from momenta that match that of the surface projections of the bulk's nodes. At the (001) surface, $\Gamma$ and $\mathrm{R}$ bulk points are maximally separated, being projected onto the $\bar{\Gamma}$ and $\bar{M}$ points, respectively. Consequently, Fermi arcs characterized by an extremely large extension appear, spanning the whole surface Brillouin zone as illustrated in Fig. 1c, with a dispersion which changes sign under a mirror operation.

Figure 1d shows a topographical overview of the $\mathrm{PdGa}(001)$ surface (experimental details on samples preparation can be found in "Methods" and Supplementary Fig. 1). Large, atomically flat terraces with extremely low defect concentrations are visible, confirming the high quality of the crystals. The line profile analysis allows the identification of the smallest step height, which is $2.5 \AA$. This value matches well with that of half the unit cell as illustrated in the inset, where adjacent terraces are labeled I and II, respectively. Atomically resolved images for crystals of opposite handedness are displayed in Fig. 1e. A square lattice is visible in both enantiomers with a periodicity $a=4.9 \AA$ matching the bulk lattice constant. A careful inspection of the perturbation developing around native defects reveals the presence of a strongly anisotropic pattern which cannot be superimposed on its mirror image. As illustrated in Supplementary Figs. 2, 3 and discussed in Supplementary Note 2, this behavior is consistent with the bulk characterization of the two enantiomers, providing a direct real space signature of the structural bulk chirality.

The local density of states (LDOS) has been experimentally inferred by scanning tunneling spectroscopy (STS) measurements. Results are reported in Fig. If. The minimum in the LDOS that is visible around the Fermi level highlights the semi-metallic character of the compound. Even though STS strongly depends on how electronic states decay into the vacuum, with higher sensitivity for states located at the center of the surface Brillouin zone ${ }^{24}$, our results are in good agreement with the theoretically calculated LDOS, obtained by projecting the bulk band structure over the surface. A one-to-one matching is evident for all of the most prominent features visible in the spectrum, i.e., the peaks 
a

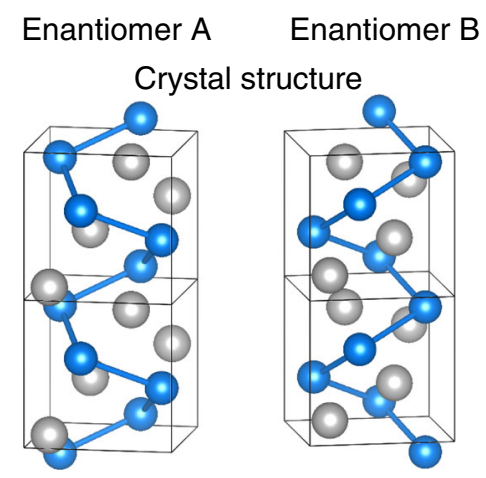

b
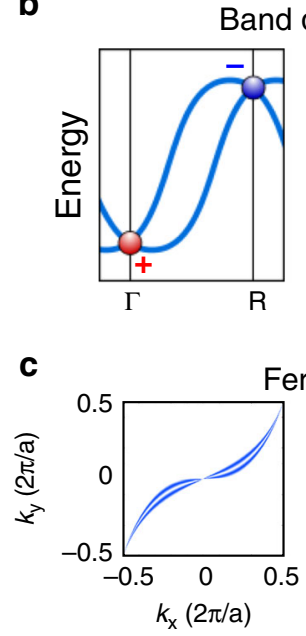

Fermi arcs

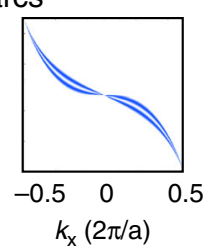

d

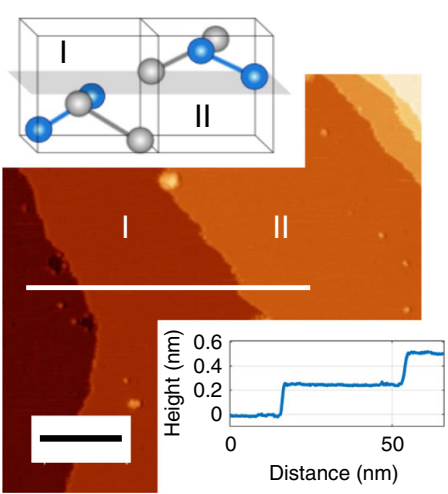

e

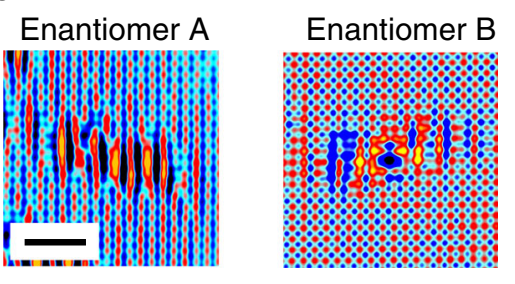

f

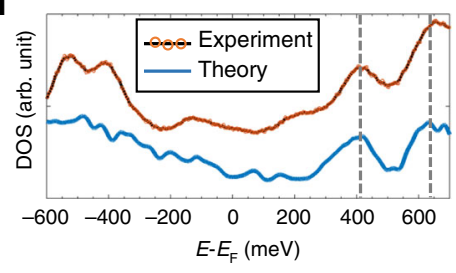

Fig. 1 Connection between chirality in real and reciprocal space in PdGa. a Crystal structure for both PdGa enantiomers. Gray and blue atoms correspond to $\mathrm{Pd}$ and $\mathrm{Ga}$, respectively. The handedness can be distinguished considering the helix formed by $\mathrm{Ga}$ atoms; $\mathbf{b}$ Schematic line cut showing the symmetryprotected band crossing at $\Gamma$ and $R$ points, respectively. The Chern number associated to the nodes reverts its sign by mirror operation. $\mathbf{c}$ Fermi arcs developing on the surface for the two different enantiomers as a result of the surface-bulk connectivity. $\mathbf{d}$ Topographic overview of the PdGa (001) surface. The line profile evidence the minimum step height, corresponding to half unit cell. The two possible surface terminations, labeled I and II, are reported in the inset. The scalebar corresponds to $20 \mathrm{~nm}$. e Atomically resolved images for crystals of opposite handedness. The perturbation developing around native defects cannot be superimposed to its mirror image. The scalebar corresponds to $5 \mathrm{~nm}$. $\mathbf{f}$ Comparison between experimental (red line) and theoretical (blue line) local density of states.

located at +400 and $+650 \mathrm{meV}$ with respect to the Fermi level (see gray dashed lines).

Handedness-dependent quasiparticle interference. To investigate how the bulk chirality impacts the electronic properties, we analyzed the standing wave patterns generated by coherent scattering of quasiparticles at defects. The resulting LDOS modulations are visualized by energy-resolved differential conductance $(\mathrm{d} I / \mathrm{d} U)$ maps that were measured at $1.9 \mathrm{~K}$ in order to have a larger coherence length and an improved energy resolution. Fourier transforms (FT) of these data allow the quantitative analysis of this information in reciprocal space, making the visualization of the scattering vectors $\mathbf{q}$ that connect the initial $\mathbf{k}_{\mathrm{i}}$ and final $\mathbf{k}_{\mathrm{f}}$ states on an iso-energy contour possible, i.e., $\mathbf{q}=\mathbf{k}_{\mathrm{i}}-\mathbf{k}_{\mathrm{f}}$. This technique, originally developed in the context of trivial surface states in noble metal surfaces ${ }^{25}$, has recently been applied to investigate the unconventional electronic properties of different classes of topologically non-trivial materials ${ }^{26-29}$. In contrast to conventional photoemission, this method allows access of both, occupied and unoccupied states, thus providing a complete spectroscopic characterization of quasiparticles that sit close to the Fermi level, i.e., those dominating the transport properties. This is particularly important in the present case. Photoemission studies have shown that Fermi arcs are overlapping with topologically trivial bulk bands for occupied states, complicating their identification ${ }^{23}$. On the other hand, theoretical calculations predict them to become strongly decoupled from bulk states at positive energies, a scenario favoring their experimental detection (see Supplementary Fig. 4).

Figure $2 \mathrm{a}-\mathrm{h}$ summarizes the results obtained at four representative energies from two $\mathrm{PdGa}(001)$ single crystals with opposite chiralities. The Bragg spots of the square (001) surface lattice can clearly be identified (highlighted by four gray circles). They are located at a distance $2 \pi / a$ from the center, with $a=$ $4.9 \mathrm{~A}$ being the lattice constant. The FT-maps show a rich plethora of scattering vectors. Their lengths, and even more remarkably their pattern, rapidly evolve with energy. No FTpattern is visible for occupied states, a direct consequence of Fermi arcs overlapping with bulk states, generating a continuum of possible scattering vectors which washes out any Fermi-arc distinct features (see Supplementary Fig. 5). For each energy, a one-to-one comparison of measurements taken on crystals of opposite handedness clearly reveals, despite different background contributions, that scattering events are chiral, i.e., FT-maps taken on the two enantiomers are related by a mirror operation. 

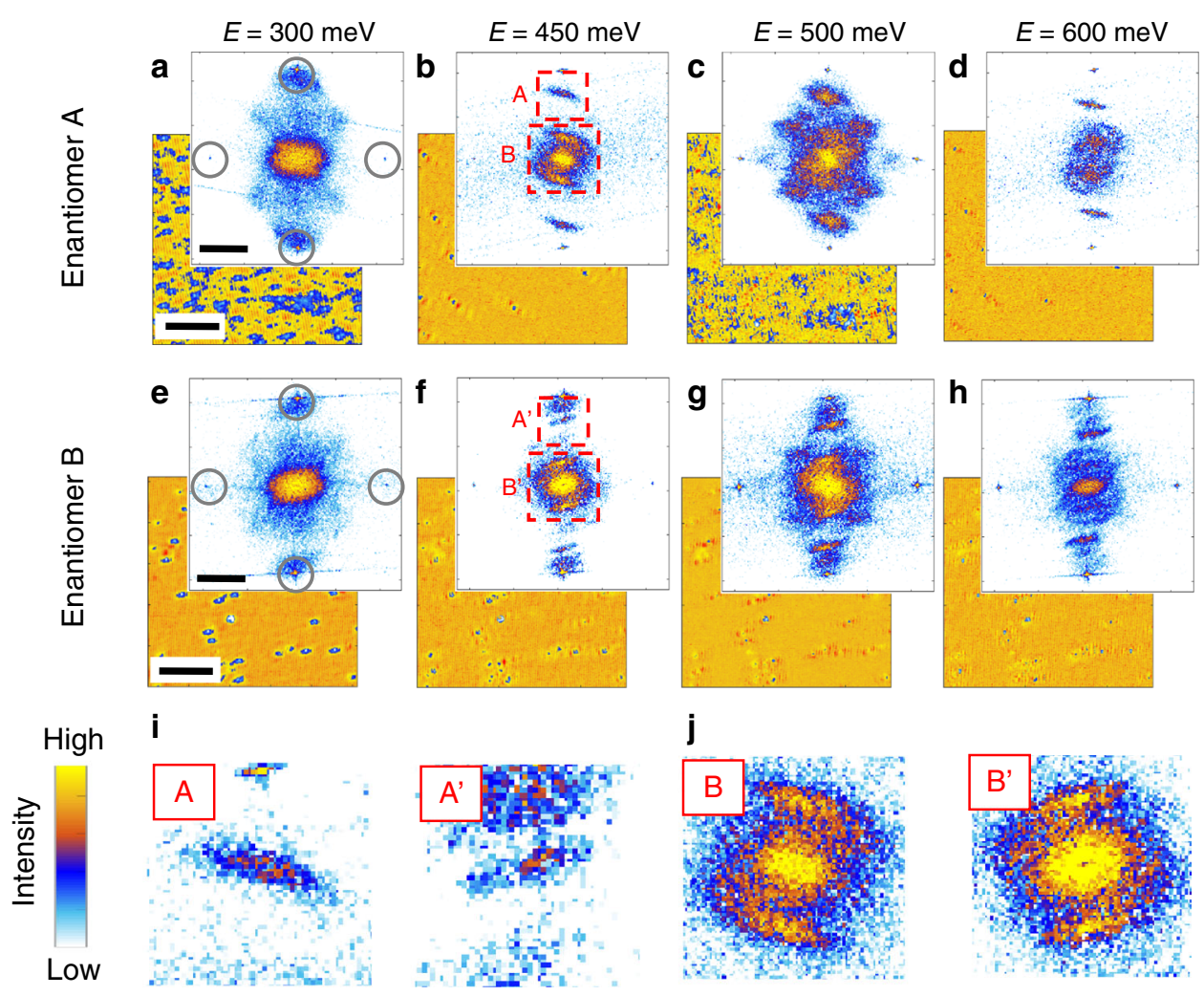

Fig. 2 Quasiparticle interference of two PdGa(001) enantiomers. a-h dl/dU maps and relative Fourier transformations obtained on $\mathrm{PdGa}(001)$ with opposite bulk chiralities at four representatives energies. Both long $A\left(A^{\prime}\right)$ and short wavelength $B\left(B^{\prime}\right)$ scattering vectors are visible, whose shapes rapidly evolve with energy. Bragg spots (see gray circles) are representatives of the surface square lattice. As highlighted in panels $\mathbf{i}$ and $\mathbf{j}$, the scattering vectors are chiral. The scalebar corresponds to $10 \mathrm{~nm}$ for $\mathrm{d} / / \mathrm{d} U$ maps and $\pi /$ a for Fourier transformations, with a being the lattice constant.

As highlighted in the zoomed FT-maps displayed in panel i and $\mathrm{j}$, this is the case for both long and short scattering vectors, labeled $\mathrm{A}\left(\mathrm{A}^{\prime}\right)$ and $\mathrm{B}\left(\mathrm{B}^{\prime}\right)$, respectively.

Measurements performed on terraces separated by half-integer unit cells provide the very same results (see Supplementary Fig. 6). This demonstrates that our findings are not related to possible terrace-dependent trivial surface states, but are an intrinsic property of the overall $\mathrm{PdGa}(001)$ surface termination. In addition, $\mathrm{Mn}$ adatoms were dosed onto the surface. Despite this procedure, which was applied to increase the disorder, resulting in a significantly stronger background in the FT-maps, large $\mathbf{q}$ scattering vectors [labeled $\mathrm{A}\left(\mathrm{A}^{\prime}\right)$ in Fig. 2] are still visible (see Supplementary Fig. 7), confirming that the signatures visible in our FT-maps are of a topological origin.

Furthermore, our measurements reveal opposite spiraling directions in the energy-dependent evolution of the QPI pattern for the two enantiomers, as illustrated in Fig. 3, where the long scattering vectors (A and A' in Fig. 2) are shown at progressively higher energies. A comparison between enantiomers $\mathrm{A}$ and $\mathrm{B}$ reveal their opposite rotational sense: anticlockwise vs. clockwise, respectively.

Theoretical analysis. The emergence of chirality-dependent scattering between topological Fermi arcs is further supported by our theoretical analysis reported in Fig. 4. Panel a shows a constant energy cut at $E=+450 \mathrm{meV}$ for both $\mathrm{PdGa}(001)$ enantiomers, showing long chiral Fermi arcs that are well decoupled from bulk states. As expected, only a mirror operation can convert one handedness of the Fermi arcs to the other. The FT-maps, calculated by including the spin-dependent scattering probabilities that take into account the influence of the relative spin orientations of the initial and final states, are displayed in
Fig. $4 \mathrm{~b}$. The FT-maps, and thus the scattering events, are chiral in the two PdGa enantiomers and are related by a mirror operation. The rich plethora of scattering vectors and their rapid evolution as a function of energy makes it difficult to establish a one-to-one correspondence for all features visible in the FT- $\mathrm{d} I / \mathrm{d} U$ maps. However, the large scattering vector [labeled $\mathrm{A}\left(\mathrm{A}^{\prime}\right)$ in Fig. 2] is sufficiently decoupled from the typical background centered around $\mathbf{q}=0$, so allowing for a detailed theoretical analysis of its origin. This is illustrated in Fig. 4c, d, where different sections of the constant energy cuts are progressively included in our analysis. The resulting FT-patterns unambiguously prove that vectors $\mathrm{A}\left(\mathrm{A}^{\prime}\right)$ are directly linked to scattering events between opposite surface Fermi arcs connected by good nesting vectors.

Energy extension of the Fermi-arcs. The existence of quantum interference patterns originating from Fermi arcs allows for their investigation as a function of energy. This is shown in Fig. 5a, which shows the experimentally obtained energy-dependent length of the scattering vector A (see Supplementary Fig. 8 for a description of the analysis procedure). These data reveal that Fermi arcs in PdGa are dominant up to $850 \mathrm{meV}$ above the Fermi level. As mentioned above, no clear information can be obtained by QPI mapping for occupied states. As complementary information, we present ARPES data of PdGa crystals in panel b which show that the band bottom of the Fermi arcs is located at approximately $780 \mathrm{meV}$ below the Fermi level (see "Methods" for a description of the measurement procedure). Overall this provides direct experimental evidence of one of the distinct features theoretically predicted for topological chiral crystals, i.e. the persistence of non-trivial Fermi arcs over a very large energy range, establishing an experimental record of more than $1.6 \mathrm{eV}$, almost symmetrically centered around the Fermi level. 


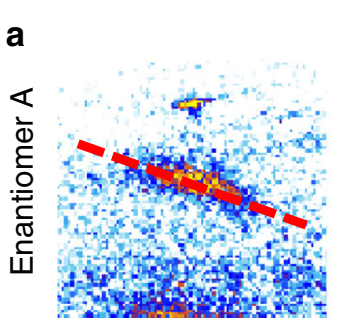

$$
E=450 \mathrm{meV}
$$

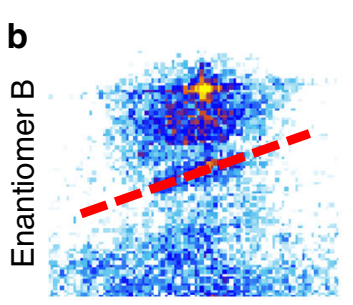

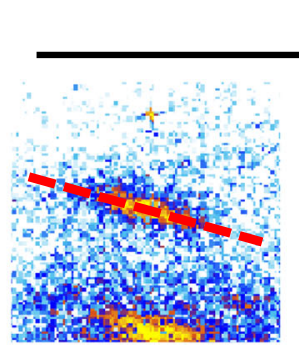

$E=500 \mathrm{meV}$

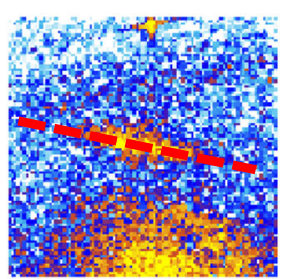

$E=600 \mathrm{meV}$

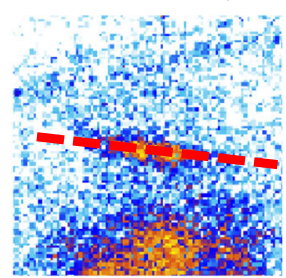

$E=700 \mathrm{meV}$

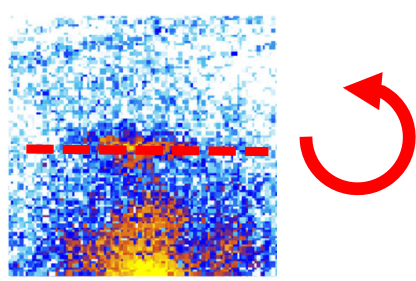

$E=800 \mathrm{meV}$
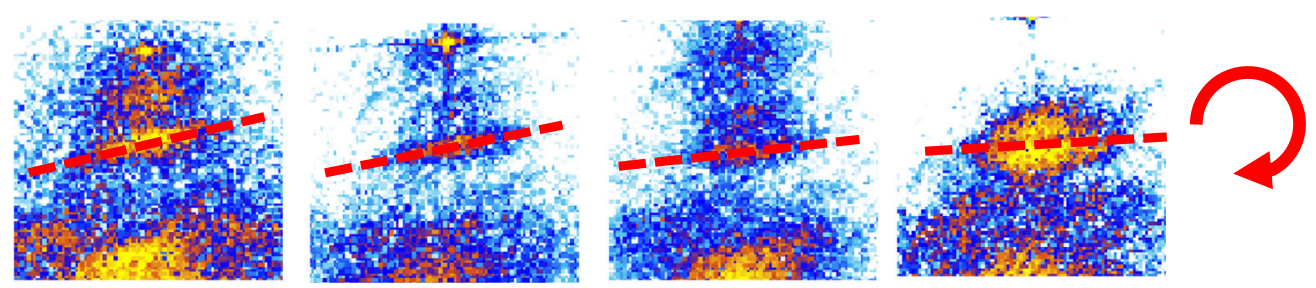

Fig. 3 Spiraling direction of QPI patterns in the two enantiomers. a, b Report the energy evolution of the long scattering vector ( $A$ and $A^{\prime}$ in Fig. 2) for enantiomers $A$ and $B$, respectively. By progressively increasing the energy, the scattering vector spirals in opposite directions, i.e., anticlockwise and clockwise, in the two enantiomers.

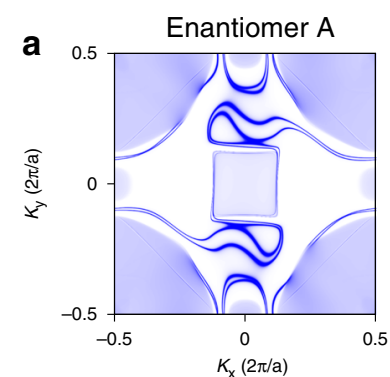

b

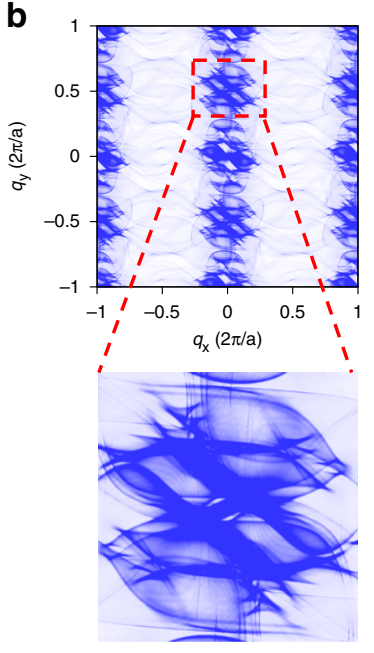

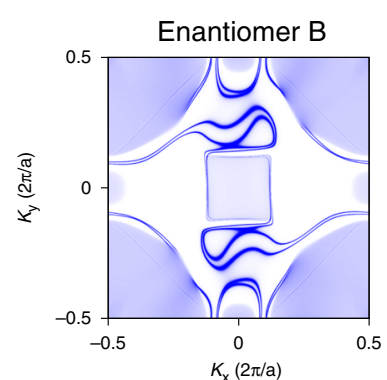

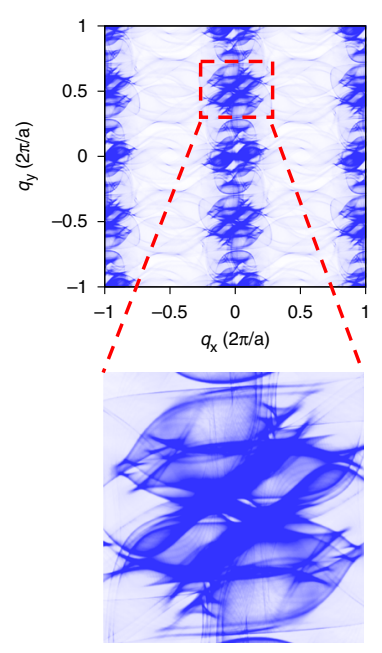

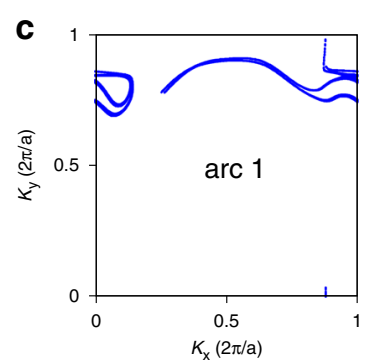
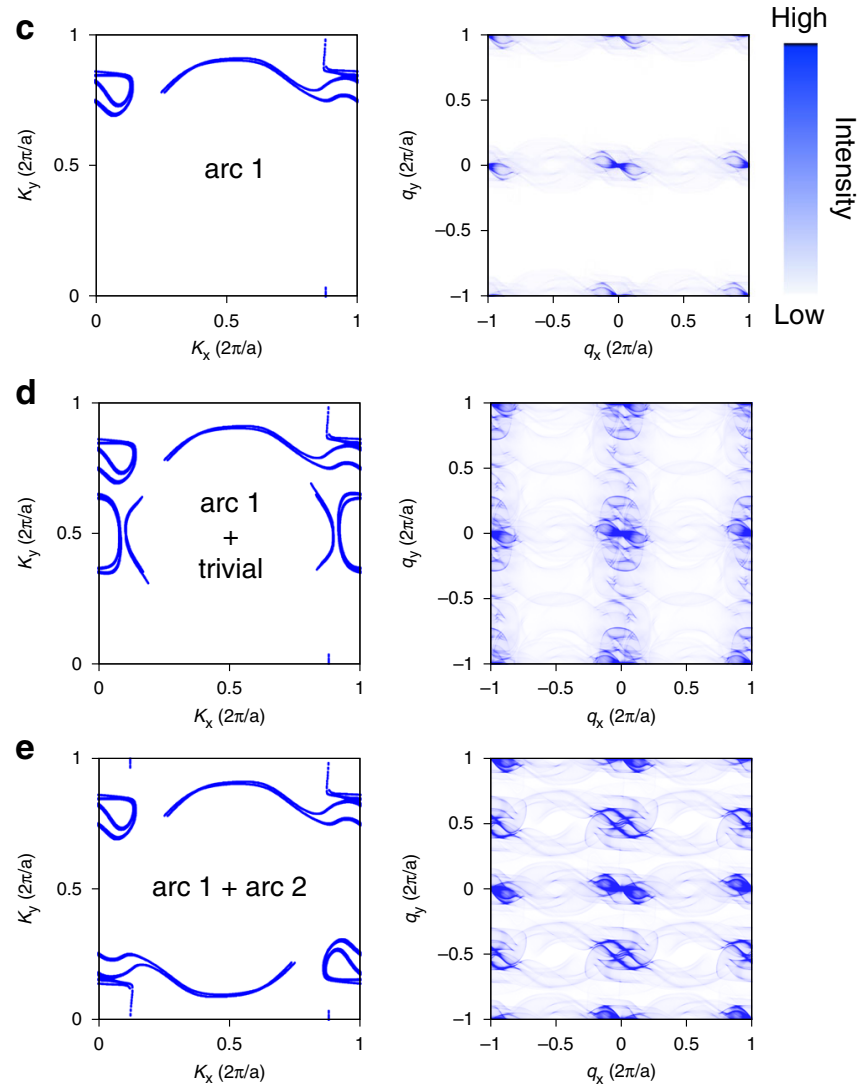

Fig. 4 Fermi arcs and chiral quasiparticle interference. a Constant energy cuts at $E=450 \mathrm{meV}$ for the two different PdGa(001) enantiomers.

b Theoretically calculated FT scattering maps. c-e constant energy cuts and their relative scattering pattern by progressively including: conly one Fermi arc; d one Fermi arc and trivial states; e all Fermi arcs.

\section{Discussion}

Our work reveals the emergence of quantum interference in topological chiral crystals that depends on the crystal enantiomer. Consequently, in this class of materials, the surface-bulk correspondence not only guarantees the existence of topologically protected surface states, but also determines how they propagate and scatter. This phenomenon directly follows from the deep connection between chirality in real and reciprocal space, and is a direct manifestation of Chern numbers changing their sign by changing the handedness of the crystal structure. These findings, jointly with the investigation of both PdGa enantiomers and the large extension of the 
a

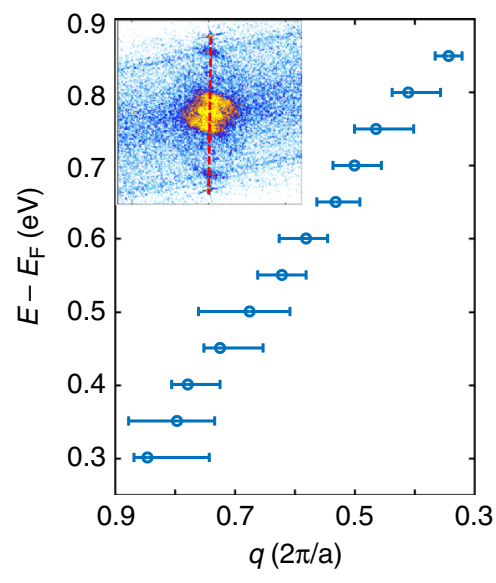

b

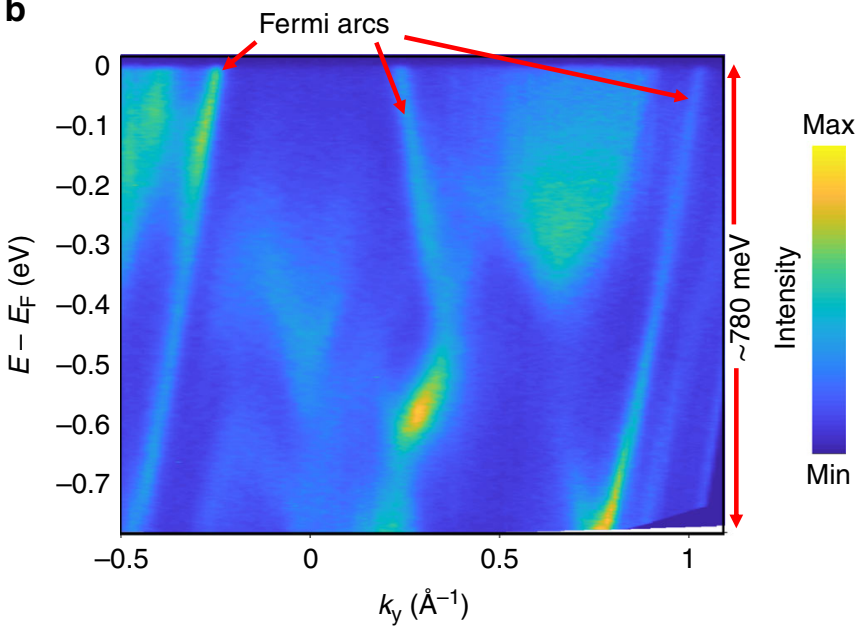

Fig. 5 Energy window for Fermi arcs. a Energy dispersion of the scattering vector associated to scattering events between opposite Fermi arcs (labeled A in Fig. 2). The wavelength has been obtained, for all energies, by analysing the intensity profile taken along the red line passing through the center of the FT-dl/dU map, as illustrated in the inset. $\mathbf{b}$ Energy dispersion of the Fermi arcs for occupied states as obtained by ARPES. The momentum direction was chosen along a path where the band bottom of the Fermi-arc becomes visible (see Supplementary Fig. 9 for details). Measurements were performed with $60 \mathrm{eV}$ photon energy and linear-horizontal polarization.

Fermi arcs, demonstrate that this material is a near ideal topological semimetal. This suggests that the topological response of $\mathrm{PdGa}$ can be accessed in transport and optical measurements ${ }^{30}$.

\section{Methods}

Sample preparation. The PdGa single crystals were grown using the self-flux technique and subsequently polished to expose the (001) surface. The atomically flat surfaces were prepared by cycles of $\mathrm{Ar}^{+}$ion sputtering and annealing in an ultra-high vacuum chamber with a base pressure of $1 \mathrm{e}-10 \mathrm{mbar}$. During the sputtering, we first turned on the titanium sublimation pump for $1 \mathrm{~min}$, then filled the chamber with $5 \mathrm{e}-5 \mathrm{mbar}$ Ar gas. The emission current and high voltage of the ion gun were set to $20 \mathrm{~mA}$ and $2.0 \mathrm{kV}$, respectively. The ion current was typically $1 \mu \mathrm{A}$. The sputtering time varies between 1 and $5 \mathrm{~h}$, depending on the condition of the surface. After sputtering, the single crystal was annealed at $680^{\circ} \mathrm{C}$ for $2 \mathrm{~h}$ by radiation heating. Supplementary Fig. 1 reports large scale topographic images (top row) and their derivative (bottom row) of a PdGa single crystal (001) surface as a function of the number of sputtering and annealing cycles. After 16 cycles, the surface shows clean terraces with a residual small number of defects. A strong accumulation of steps is visible. Atomically flat terraces with a width of approximately $50 \mathrm{~nm}$ are present in our samples. These are used for the quasiparticle interference experiments described in the main text.

STM measurements. Low-temperature STM measurements were performed using a cryostat (Oxford Instruments) equipped with an UHV insert hosting a Tribus STM head (Sigma Surface Science) operated at a temperature $T=1.9 \mathrm{~K}$. All measurements have been performed using electrochemically etched tungsten tips. Before measurements, the tips were conditioned on a $\mathrm{Ag}(111)$ single crystal. Spectroscopic data have been obtained using the lock-in technique and a bias voltage modulation in between 1 and $20 \mathrm{meV}$ at a frequency of $793 \mathrm{~Hz}$, with the amplitude progressively increasing with the scanning bias. $\mathrm{dI} / \mathrm{dU}$ maps have been acquired simultaneously to topographic images.

ARPES measurements. The ARPES experiments were performed at the highresolution ARPES branch line of the beamline I05 at the Diamond Light Source, UK, with a Scienta R4000 analyzer at a temperature below $20 \mathrm{~K}$. The momentum direction of the band dispersion shown in Fig. $5 \mathrm{~b}$ of the main manuscript is indicated by the red arrow in Supplementary Fig. 9. The line-cut was chosen at a position where the bottom of the Fermi-arc was at the largest binding energy in the surface Brillouin zone.

\section{Data availability}

The data supporting the findings of this study are available from the corresponding authors upon reasonable request.
Received: 10 May 2020; Accepted: 16 June 2020;

Published online: 14 July 2020

\section{References}

1. Hasan, M. Z. \& Kane, C. L. Colloquium: topological insulators. Rev. Mod. Phys. 82, 3045-3067 (2010).

2. Qi, X.-L. \& Zhang, S.-C. Toplogical insulators and superconductors. Rev. Mod. Phys. 83, 1057-1110 (2011).

3. Mourik, V. et al. Signatures of Majorana fermions in hybrid superconductor semiconductor hybrid devices. Science 336, 1003-1007 (2012).

4. Nadj-Perge, S. et al. Observation of Majorana fermions in ferromagnetic atomic chains on a superconductor. Science 346, 602-607 (2014).

5. $\mathrm{Xu}, \mathrm{S}$.-Y. et al. Discovery of a Weyl femion semimetal and topological Fermi arcs. Science 349, 613-617 (2015).

6. Lv, B. Q. et al. Experimental discovery of Weyl semimetal TaAs. Phys. Rev. X 5, 031013 (2015).

7. Bradlyn, B. et al. Beyond Dirac and Weyl fermions: unconventional quasiparticles in conventional crystals. Science 353, 5037 (2016).

8. Ezawa, M. Pseudospin $3 / 2$ fermions, type-II Weyl semimetals, and critical Weyl semimetals in tricolor cubic lattices. Phys. Rev. B 94, 195205 (2016).

9. $\quad$ Liang, L. \& Yu, Y. Semimetal with both Rarita-Schwinger-Weyl and Weyl excitations. Phys. Rev. B 93, 045113 (2016).

10. Tang, P., Zhou, Q. \& Zhang, S.-C. Multiple types of topological fermions in transition metal silicides. Phys. Rev. Lett. 119, 206402 (2017).

11. Chang, G. et al. Topological quantum properties of chiral crystals. Nat. Mater 17, 978-985 (2018).

12. Mühlbauer, S. et al. Skyrmion lattice in a chiral magnet. Science 323, 915-919 (2009).

13. Rikken, G. L. J. A. \& Raupach, E. Observation of magneto-chiral dichroism. Nature 390, 493-494 (1997)

14. Carnicom, E. M. et al. $\mathrm{TaRh}_{2} \mathrm{~B}_{2}$ and $\mathrm{NbRh}_{2} \mathrm{~B}_{2}$ : Superconductors with a chiral noncentrosymmetric crystal structure. Sci. Adv. https://doi.org/10.1126/sciadv. aar7969 (2018).

15. de Juan, F. et al. Quantized circular photogalvanic effect in Weyl semimetals. Nat. Commun. 8, 15995 (2017)

16. Rinkel, P., Lopes, P. L. S. \& Garate, I. Signatures of the chiral anomaly in phonon dynamics. Phys. Rev. Lett. 119, 107401 (2017).

17. Zhong, S., Moore, J. E. \& Souza, I. Gyrotropic magnetic effect and the magnetic moment on the Fermi surface. Phys. Rev. Lett. 116, 077201 (2016)

18. Sanchez, D. S. et al. Topological chiral crystals with helicoid-arc quantum states. Nature 567, 500-505 (2019).

19. Schröter, N. B. M. et al. Chiral topological semimetal with multifold band crossings and long Fermi arcs. Nat. Phys. 15, 759-765 (2019). 
20. Yuan, Q.-Q. et al. Quasiparticle interference evidence of the topological Fermi arc states in chiral fermionic semimetal CoSi. Sci. Adv. https://doi.org/ 10.1126/sciadv.aaw9485 (2019).

21. Li, H. et al. Chiral fermion reversal in chiral crystals. Nat. Commun. 10, 5505 (2019).

22. Yao, M. et al. Observation of giant spin-split Fermi-arc with maximal Chern number in the chiral topological semimetal PtGa. Nat. Commun. 11, 2033 (2020).

23. Schröter, N. B. M. et al. Observation and manipulation of maximal Chern numbers in the chiral topological semimetals PdGa. Preprint at http://arxiv. org/abs/1907.08723 (2019).

24. Stroscio, J. A. et al. Tunneling spectroscopy of bcc (001) surface states. Phys. Rev. Lett. 75, 2960-2963 (1995).

25. Crommie, M. F., Lutz, C. P. \& Eigler, D. M. Imaging standing waves in a twodimensional electron gas. Nature 363, 524-527 (1993).

26. Roushan, P. et al. Topological surface states protected from backscattering by chiral spin texture. Nature 460, 1106-1109 (2009).

27. Zhang, T. et al. Experimental demonstration of topological surface states protected by time-reversal symmetry. Phys. Rev. Lett. 103, 266803 (2009)

28. Okada, Y. et al. Observation of Dirac node formation and mass acquisition in a topological crystalline insulator. Science 341, 1496-1499 (2013).

29. Inoue, $\mathrm{H}$. et al. Quasiparticle interference of the Fermi arcs and surface-bulk connectivity of a Weyl semimetal. Science 351, 1184-1187 (2016).

30. Chang, G. et al. Unconventional photocurrents from surface Fermi arcs in topological chiral semimetals. Phys. Rev. Lett. 124, 166404 (2020).

\section{Acknowledgements}

The work in MPI-MSP was financially supported by the Deutsche Forschungsgemeinschaft (DFG, German Research Foundation)_Project number 314790414. The work in MPI-CPFS Dresden was financially supported by the European Research Council (ERC) Advanced Grant No. 742068 "TOP-MAT” and the WürzburgDresden Cluster of Excellence on Complexity and Topology in Quantum Matter ct.qmat (EXC2147, project ID 39085490). N.B.M.S. was supported by Microsoft. The contribution from Empa was financially supported by the Swiss National Science Foundation (SNF) - project number 159690

\section{Author contributions}

K.M. grew the PdGa single crystals and analyzed their crystal structure with the help of H.B. P.S., F.K., J.-R.J., and K.C performed and analyzed the STM measurements.
N.B.M.S., S.S., J.A.K., D.P, T.K.K., P.D., C.C., R.W., and W.S. performed and analyzed the ARPES measurements. The theoretical calculations and analysis were performed by F.-R.F. and Y.S. P.S., F.K., K.M., K.C., Y.S., and S.S.P.P. wrote the manuscript. All coauthors read and commented on the manuscript. The work was guided by P.S., K.M., Y.S., C.F., and S.P.P.S.

\section{Competing interests}

The authors declare no competing interests.

\section{Additional information}

Supplementary information is available for this paper at https://doi.org/10.1038/s41467020-17261-x.

Correspondence and requests for materials should be addressed to P.S. or S.S.P.P.

Peer review information Nature Communications thanks the anonymous reviewer(s) for their contribution to the peer review of this work.

Reprints and permission information is available at http://www.nature.com/reprints

Publisher's note Springer Nature remains neutral with regard to jurisdictional claims in published maps and institutional affiliations.
Open Access This article is licensed under a Creative Commons Attribution 4.0 International License, which permits use, sharing, adaptation, distribution and reproduction in any medium or format, as long as you give appropriate credit to the original author(s) and the source, provide a link to the Creative Commons license, and indicate if changes were made. The images or other third party material in this article are included in the article's Creative Commons license, unless indicated otherwise in a credit line to the material. If material is not included in the article's Creative Commons license and your intended use is not permitted by statutory regulation or exceeds the permitted use, you will need to obtain permission directly from the copyright holder. To view a copy of this license, visit http://creativecommons.org/ licenses/by/4.0/.

(C) The Author(s) 2020 